Article

\title{
The Role of the Effective Cloud Albedo for Climate Monitoring and Analysis
}

\author{
Richard Mueller $^{1, \star}$, Jörg Trentmann ${ }^{1}$, Christine Träger-Chatterjee ${ }^{1}$, Rebekka Posselt ${ }^{2}$ and \\ Reto Stöckli $^{2}$
}

${ }^{1}$ Deutscher Wetterdienst, Frankfurter Str. 135, D-60387 Offenbach, Germany;

E-Mails: joerg.trentmann@dwd.de (J.T.); Christine.Traeger-Chatterjee@dwd.de (C.T.-C.)

${ }^{2}$ MeteoSchweiz, Kräbülstr. 58, CH-8044 Zürich, Switzerland;

E-Mails: rebekka.posselt@meteoswiss.ch (R.P.); reto.stoeckli@meteoswiss.ch (R.S.)

* Author to whom correspondence should be addressed; E-Mail: richard.mueller@dwd.de;

Tel.: +49-69-8062-4922; Fax: +49-69-8062-3759.

Received: 24 August 2011; in revised form: 13 October 2011 / Accepted: 13 October 2011 /

Published: 25 October 2011

\begin{abstract}
Cloud properties and the Earth's radiation budget are defined as essential climate variables by the Global Climate Observing System (GCOS). The cloud albedo is a measure for the portion of solar radiation reflected back to space by clouds. This information is essential for the analysis and interpretation of the Earth's radiation budget and the solar surface irradiance. We present and discuss a method for the production of the effective cloud albedo and the solar surface irradiance based on the visible channel $(0.45-1 \mu \mathrm{m})$ on-board of the Meteosat satellites. This method includes a newly developed self-calibration approach and has been used to generate a 23-year long (1983-2005) continuous and validated climate data record of the effective cloud albedo and the solar surface irradiance. Using this climate data record we demonstrate the ability of the method to generate the two essential climate variables in high accuracy and homogeneity. Further on, we discuss the role of the cloud albedo within climate monitoring and analysis. We found trends with opposite sign in the observed effective cloud albedo resulting in positive trends in the solar surface irradiance over ocean and partly negative trends over land. Ground measurements are scarce over the ocean and thus satellite-derived effective cloud albedo and solar surface irradiance constitutes a unique observational data source. Within this scope it has to be considered that the ocean is the main energy reservoir of the Earth, which emphasises the role of
\end{abstract}


satellite-observed effective cloud albedo and derived solar surface irradiance as essential climate variables for climate monitoring and analysis.

Keywords: cloud albedo; solar irradiance; Earth radiation budget; climate

\section{Introduction}

Clouds play a dominant role within the Earth's radiation budget [1-4]. The warming effect induced by the increase of greenhouse gases might be modified by changes in the Earth's albedo on a regional or global scale. Especially changes in the variability, but also extremes of the cloud albedo could lead to a substantial change of the planetary albedo. Without clouds the Earth's albedo would be halved. The reflected solar energy would drop from $\sim 100$ to $\sim 50 \mathrm{~W} / \mathrm{m}^{2}$ and the absorbed solar energy would increase from $\sim 240$ to $\sim 290 \mathrm{~W} / \mathrm{m}^{2}[2,4]$. In a non-cloud Earth this effect could only partly be compensated by an increase of the emitted thermal radiation. On average, clouds have a net cooling effect, as illustrated in Figure 1.

Figure 1. Net cooling induced by the global cloud field as given by the NCEP reanalysis data. The net cooling is especially pronounced in the equatorial belt. In the polar regions the planetary albedo is not increased by clouds as a consequence of the large surface albedo. Here, the "warming" effect of clouds in the thermal spectra dominates.

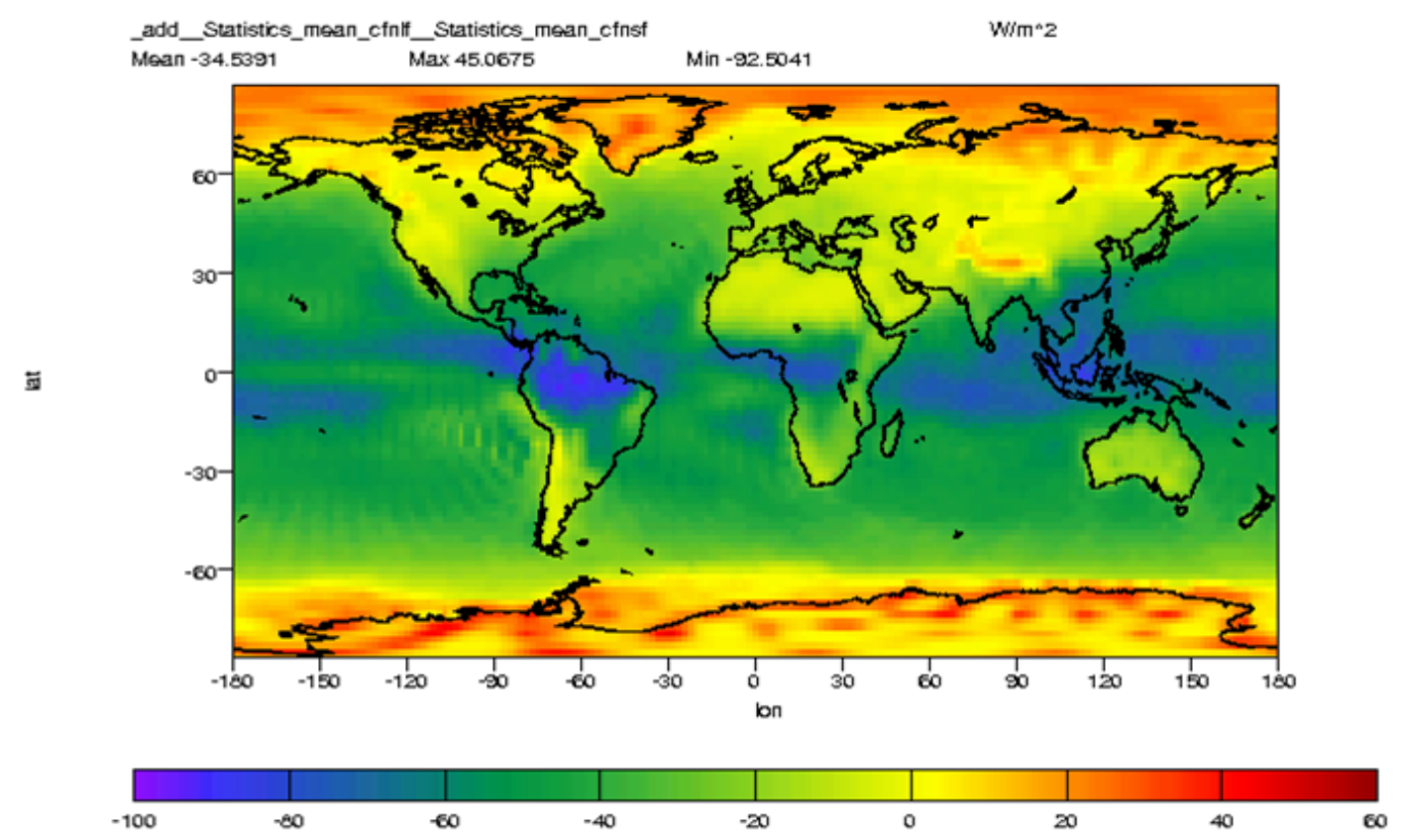

The net cooling effect of clouds is approximately 10 times higher than the decadal anthropogenic radiative forcing induced by increase of greenhouse gases. Trends and changes in extremes of the cloud albedo would change the cooling effect of clouds and would therefore significantly affect the climate on a global and regional scale. Hence, the effective cloud albedo plays a dominant role in the Earth's 
radiation and energy budget. Furthermore, this quantity can be observed from satellites without the need of any model or otherwise external data source.

The established satellite based data sets of the International Satellite Cloud Climatology Project (ISCCP) [5] and the Global Energy and Water Cycle Experiment (GEWEX) [6] does not offer the effective cloud albedo. It is also not offered by the ESA Climate Change Initiative. Instead, large efforts went into the monitoring and analysis of specific cloud properties (e.g., [3,7,8] and references therein), where the cloud fraction, cloud optical depth (cloud liquid water path) and cloud top height have been essential variables of interest. However, data sets of cloud fraction, cloud optical depth and cloud top height which are derived from different satellites and with different retrieval methods reveal a wide dispersion of the respective variables (http://climserv.ipsl.polytechnique.fr/gewexca/) [9] This indicates that the generation of consistent data sets is still a challenge in science that can not easily be met. The observed radiances are affected by the cloud fraction, the cloud optical depth and the cloud particle size, hence no direct function of these variables with the observed radiances is given. We believe that the effective cloud albedo and the derived solar surface irradiance provides a potential to overcome these limitations. This will be discussed in the following sections. Within this scope this publication also aims to increase the awareness of the effective cloud albedo within the climate community.

The planetary albedo, also expressed as Earth's albedo, has been subject of intensive research (e.g, [2,10]). For the Earth's albedo clouds play a quite important role, and the cloud albedo is frequently discussed within the scope of the planetary albedo and the cloud radiative forcing (e.g., [3]), as a consequence. However, the cloud albedo is a somehow theoretical quantity. Identical clouds have per definition identical cloud albedos. However, the effect of the same cloud (identical cloud) on the planetary albedo depends on the underlying surface albedo. The cloud albedo is therefore not a space observable but has to be derived from other quantities, e.g., the mirco-physical cloud properties or the effective cloud albedo as discussed in [3]. Indeed, it is not the cloud albedo but the effective cloud albedo (defined in Section 2.1) that can be directly observed from space (satellite). It provides a first estimate of the effect of clouds on the planetary albedo relative to the clear sky situation. It is therefore a measure for the cloud effect on the Earth's radiation budget, e.g., a water cloud with medium cloud optical depth over fresh snow would lead at most to a small increase of the planetary albedo while the same cloud would lead to a large increase of the planetary albedo over the ocean.

Satellite observations enable the retrieval of the effective cloud albedo with high spatial and temporal resolution and a large areal coverage (up to global). Through radiometric measurements satellite sensors provide information on the reflection of solar irradiance caused by clouds, the Earth's clear sky atmosphere and the surface. This information is the basis for the retrieval of the effective cloud albedo.

The effective cloud albedo is defined in straightforward manner by the observed reflections and exhibit the potential to generate multi-satellite data sets with high consistency and homogeneity, covering several decades. This in turn is a basis for the generation of solar surface irradiance covering the complete geostationary ring; an important task for climate monitoring and solar energy planning. Large spectral differences in the used visible channels, however, affect the consistency and homogeneity of the effective cloud albedo significantly, as discussed in detail in [11] and should be avoided for the derivation of the effective cloud albedo, therefore. 
In order to discuss the role of the cloud albedo for climate monitoring and analysis a 23-year long (1983-2005) continuous and validated data set of effective cloud albedo (CAL) based on the broadband visible channel $(0.45-1 \mu \mathrm{m})$ of the MVIRI instruments on-board of the Meteosat First Generation (MFG) satellites is used.

The CAL data set is generated with the climate version of the Heliosat algorithm [12,13], which includes a self-calibration method and an improved algorithm for the determination of the clear-sky reflectivity. In Section 2.3 the self-calibration method is described and validation results are presented. In order to provide an operational satellite service Eumetsat's first generation geostationary satellites had to be regularly replaced. The purpose of the self-calibration method is to automatically account for the degradation of the individual satellite instruments during their lifetime and the discontinuities induced by the launch of MVIRI instruments with identical design but different brightness sensitivity. The self-calibration enables the generation of a temporal homogeneous time series of the effective cloud albedo and the subsequently derived solar surface irradiance.

\section{Method and Data}

\subsection{The Heliosat Method}

The effective cloud albedo is derived from the geostationary satellite by the use of the observed reflections in the visible broadband channel, without the need of any further information. Instead of radiance the Heliosat method can be applied directly to the observed reflection given in digital counts to determine the effective cloud albedo, also denoted cloud index by other authors [12-14]. In this way, it neither depends on any calibration information nor on information from other channels.

The Heliosat method uses the level 1.5 satellite data of the Earth reflection containing rectified digital pixel counts of the High Resolution Visible (HRV) channel from Meteosat First Generation satellites. The respective data are called "Rectified Image Data" and provided by Eumetsat [15]. No information from other channels is used.

The satellites were located over the equator at a longitude of $0^{0}$. The field of view of the satellites reaches up to $80^{\circ} \mathrm{N} / \mathrm{S}$ and $80^{\circ} \mathrm{E} / \mathrm{W}$, respectively and is illustrated in Figure 2 using clear sky reflection and effective cloud albedo images. The MFG satellites (Meteosat 2-7, in operation 1982-2005) carried the Meteosat Visible and InfraRed Imager (MVIRI), a radiometer with 3 spectral bands in the visible band (VISSN: $0.45-1 \mu \mathrm{m}$ ), in the water vapor band (WV: 5.7-7.1 $\mu \mathrm{m}$ ) and infrared band (IR: 5.7-7.1 $\mu \mathrm{m}$ ). One scan of the visible disc was accomplished within $30 \mathrm{~min}$ at a horizontal resolution of around $2.5 \mathrm{~km}$ at the sub-satellite point. The instrumental changes between the Meteosat Visible and Infrared Imager (MVIRI) instruments 2 to 7 on the first generation of Meteosat satellites between 1983 and 2005 were minor. In particular, all instruments measured the reflectance in the same spectral band in the visible wavelength region of $0.45-1 \mu \mathrm{m}$ (VISSN). For more information on MVIRI refer to the EUMETSAT homepage [16].

The effective cloud albedo is defined as the normalised difference between the all sky and clear sky reflection in the visible observed by the satellite. The deviation of the all sky reflection of the pixel from the clear sky reflection is a measure of the cloud effect on the Earth's solar radiation budget, hence, a measure for the cooling effect of clouds in the solar spectrum. For example, the larger the effective cloud 
albedo over ocean and vegetation, the larger the "solar cooling effect". In order to derive the effective cloud albedo different luminance conditions arising from variations in the observed reflections due the Sun-Earth distance and the solar zenith angle have to be corrected. Furthermore, the dark offset of the instrument has to be subtracted from the satellite image counts. The observed reflections are therefore normalised by application of Equation (1):

$$
\rho=\frac{D-D_{0}}{f \cos (\theta)}
$$

Here, $\mathrm{D}$ is the observed digital count including the dark offset of the satellite instrument. $D_{0}$ is the dark offset, which is the baseline value of the instrument in the absence of irradiance and has to be subtracted. The sun-earth distance variation is taken into account by the factor f. Finally, the cosine of the solar zenith angle corrects the different illumination conditions at the top of atmosphere introduced by different solar altitudes

The effective cloud albedo is then derived from the normalised pixel reflection $\rho$, the clear sky reflection $\rho_{c s}$ and the maximal cloud reflection $\rho_{\max }$ by Equation (2):

$$
C A L=\frac{\rho-\rho_{c s}}{\rho_{\max }-\rho_{c s}}
$$

Here, $\rho$ is the observed reflection for each pixel and time. $\rho_{c s}$ is the clear sky reflection, which is a monthly value derived for every pixel and time slot separately. This is essentially done by using the reflection of the pixel in a cloud free case. This is usually the lowest occurring reflection during a certain time span (e.g., a month) derived for each pixel of the satellite image. Further details on the method to derive $\rho_{c s}$ are given in [11].

$\rho_{\max }$ is the "maximum" reflection. It is determined by the 95th percentile of all reflection values at local noon in a target region, characterised by high frequency of cloud occurrence for each month. In this manner changes in the satellite brightness sensitivity are accounted for.

Figure 2. Example of a $\rho_{\min }$ image and and effective cloud albedo for a 11 UTC slot in June 2005.
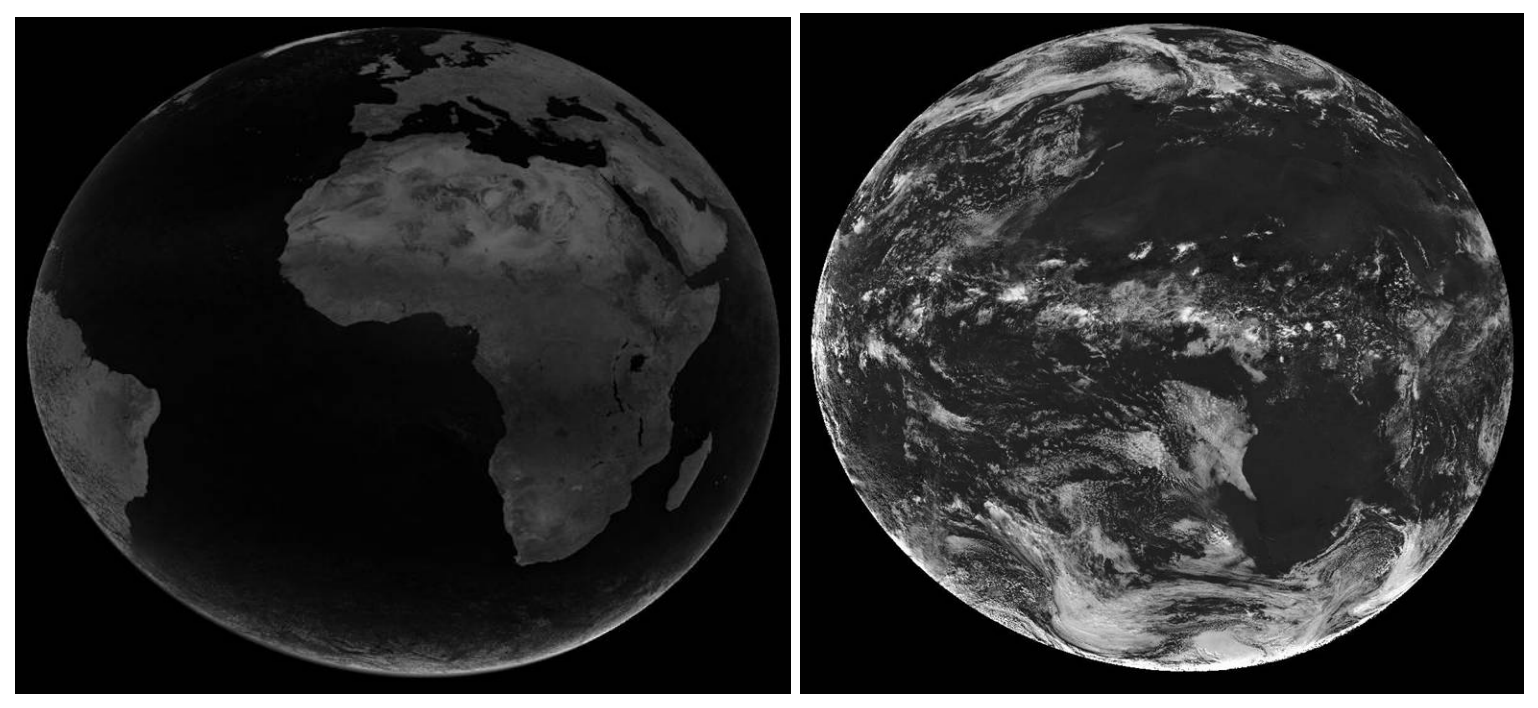
Only the observed reflections are needed to derive the effective cloud albedo by application of Equation (2). As a result the effective cloud albedo is completely defined by the satellite observation with only one broadband visible channel needed. This means that it does not rely on any external information of surface albedo or clear sky reflections.

\subsection{Relation of Effective Cloud Albedo to Solar Radiation Forcing}

The solar surface irradiance (SSI) is retrieved using the well established Heliosat relation between the effective cloud albedo and the solar irradiance [12,14], which is based on the law of energy conservation. As a consequence the basic relation between the solar irradiance and the effective cloud albedo is pre-dominantly a linear relation given in Equation (3).

$$
S S I=S S I_{c s} *(1-C A L)
$$

Here, $S S I$ is the solar surface irradiance, $S S I_{c s}$ is the clear sky surface irradiance, which is derived with the clear sky model MAGIC [17] and CAL is the effective cloud albedo, also called cloud index $\mathrm{n}$ in former publications [12]. For effective cloud albedo values above 0.8 the above equation is modified in order to consider the saturation and absorption effects within optically thick clouds. The modification of the equation for small and large values of CAL is based on ground measurements and is described in detail in [14].

Equation (3) shows that trends (changes) in CAL would transform into SSI trends (changes). In cases where the clear sky atmospheric variables are kept constant, any trends (changes) in $C A L$ transforms directly and linearly into solar forcing of SSI. As CAL can hardly be validated directly, SSI validation results can be used to evaluate the stability and climate quality of CAL. Validation of SSI has been performed with ground measurements from the Baseline of Surface Radiation Network (BSRN). BSRN is a project of the World Climate Research Program (WCRP) and the Global Energy and Water Experiment (GEWEX). The aim of the BSRN is to provide consistent quality-controlled surface radiation data from a global station network using a defined set of instrumentation and measurement protocols. The data is available free of charge at BSRN web page (www.bsrn.awi.de). The accuracy of the shortwave measurement is estimated to be $5 \mathrm{~W} / \mathrm{m}^{2}$ [18]. The surface data of all available stations that are within the full disc of the Meteosat satellite for covered period (see Figure 3) has been used for the validation. Table 1 provides the results of the SSI validation results of the CM-SAF climate data set. In addition, for the same stations the validation results of the Global Energy and Water Cycle Experiment ( GEWEX) [6,19] and the International Satellite Cloud Climatology Project (ISCCP) $[5,7,19]$ are provided. The ISCCP solar irradiance has been estimated by the use of ISCCP cloud fractional cover and optical depth. The validation results demonstrates the quality of the SSI data set and in turn of $C A L$ as it is the pre-dominant atmospheric variable defining SSI.

The above mentioned validation results transforms to an overall accuracy of 0.05 for monthly means ( $90 \%$ of cases) of cloud albedo by application of error propagation on Equation (3). More details of the validation are given in the CM-SAF validation report (www.cmsaf.eu). However, CAL can only be retrieved accurately if a certain number of clear sky cases are present within each month. In some regions and seasons, this is not always the case. In regions and periods with long-lasting clouds higher 
uncertainties occur. In such cases cloud contamination of $\rho_{c s}$ leads to artificially lower values of CAL, hence to significant errors. This artefact occurs pre-dominantly for slant geometries (border of Meteosat coverage, or wintertime above a latitude of $\pm 60^{\circ}$ ). It is expected that the accuracy of 0.05 for monthly means is not met any more in those regions and higher uncertainties might occur.

Figure 3. Shortcuts and location of the stations used for the validation. The colour gives the altitude of the land surface in meter.

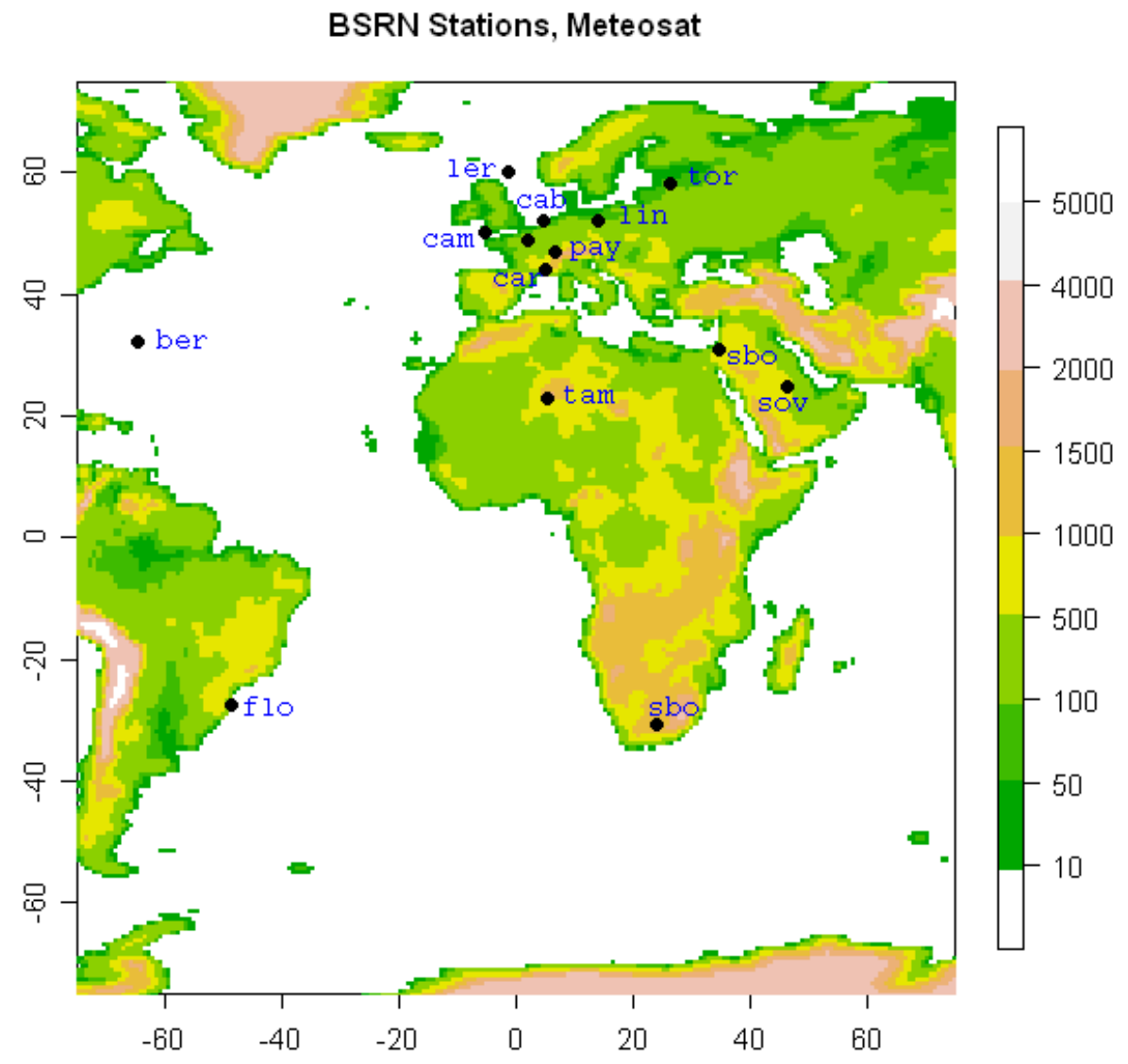

Table 1. Statistics for the comparison of monthly mean SSI between the mean of all BSRN stations and CM SAF as well as GEWEX and ISCCP. MAD is the mean difference gained as average over the absolute bias values, SD is the standard deviation, AC is the anomaly correlation and the last column gives the number of monthly mean with a bias higher than $15 \mathrm{~W} / \mathrm{m}^{2}$, a threshold resulting from the CM-SAF target accuracy of $10 \mathrm{~W} / \mathrm{m}^{2}$ plus the uncertainty of the ground measurements.

\begin{tabular}{lcccccc}
\hline $\begin{array}{l}\text { SSI } \\
\text { (monthly mean) }\end{array}$ & $\mathrm{n}_{\text {mon }}$ & $\begin{array}{c}\text { Bias } \\
{\left[\mathrm{W} / \mathrm{m}^{2}\right]}\end{array}$ & $\begin{array}{c}\text { MAD } \\
{\left[\mathrm{W} / \mathrm{m}^{2}\right]}\end{array}$ & $\begin{array}{c}\text { SD } \\
{\left[\mathrm{W} / \mathrm{m}^{2}\right]}\end{array}$ & $\mathrm{AC}$ & $\begin{array}{c}\text { Frac }_{\text {mon }}>15 \mathrm{~W} / \mathrm{m}^{2} \\
{[\%]}\end{array}$ \\
\hline \hline CM SAF-BSRN & 855 & 4.40 & 7.99 & 8.14 & 0.89 & 11.35 \\
GEWEX-BSRN & 855 & -2.56 & 12.26 & 11.12 & 0.82 & 32.75 \\
ISCCP-BSRN & 855 & -0.03 & 11.79 & 11.37 & 0.78 & 29.94 \\
\hline
\end{tabular}




\subsection{Self-Calibration}

Meteosat First Generation (MFG) satellites have been designed for now-casting purposes. The ability to retrieve climate information has been not the main focus of the MFG satellite service. As a consequence, the MVIRI instruments on-board of Meteosat First Generation satellites do not provide well calibrated radiances of the observed reflections. In order to analyse changes (trends and extremes) of the effective cloud albedo, a long time series covering several decades is needed. Hence, it is necessary to develop and apply a method which corrects for degradation (ageing) of the satellite instruments and steps induced by instrument replacement or instrument gain changes. The self-calibration method discussed in the following is designed to perform the necessary corrections.

The self-calibration algorithm is based on the determination of the "maximum" effective cloud albedo $\rho_{\max }$. Input are the sun zenith angle normalised reflections corresponding to Equation (1). In analogy to [20] a histogram of all available reflection counts is generated. However, instead of using an upper and a lower bound, the 95th percentile is used as self-calibration parameter and set to $\rho_{\max }$ following the approach of [14] and references therein. The generation of the histogram for the full disk is computational quite expensive due to the large pixel amount and does no improve the statistical stability of the 95th percentile. Thus, a the histogram is only generated for selected sub-region located in the southern Atlantic ocean between $15^{\circ} \mathrm{W}$ and $0^{\circ} \mathrm{W}$ and $58^{\circ} \mathrm{S}$ and $48^{\circ} \mathrm{S}$. This region features a high abundance of frontal systems with large cloud amounts most of the time, but hardly any convection and provides a very stable statistics. A range of percentiles has been tested and the 95 th percentile has been found to provide the best statistical stability.

The self-calibration algorithm is applied to the 1300 UTC slot, which is closest to the local noon of the region. The histogram is generated for every month using all $\rho$ values within this region. The resulting $\rho_{\text {max }}$ is then applied in the calculation of all $C A L$ values within that month, following Equation (2).

The stability and performance of the self-calibration has been evaluated against ground measurements from the Baseline Surface Radiation Network (BSRN) [18], using the solar irradiance derived with the effective cloud albedo by application of Equation (3). Here, we use the surface data of all available stations that are within the full disc of the Meteosat satellite for the covered period (see Figure 3) as a reference to evaluate the stability of the self-calibration.

Figure 4 shows the temporal evolution of the normalised bias between the CM SAF data set and the BSRN data. The normalised bias is calculated as follows: The arithmetic average over the complete time series of the BSRN and the satellite data is calculated for each station. The resulting mean difference (bias) is subtracted from all monthly means of the satellite data for each station. The mean difference (bias) over the covered time period is zero; however, trends induced by temporal changes in the monthly differences are not affected by this normalisation procedure. The differences between the monthly means of the BSRN and satellite data are then averaged over all available stations, whenever monthly means from at least 3 stations are available. This lead to an overall time series of "normalised" differences of monthly means, referred to as normalised bias. This time series is then analysed for temporal trends. A trend in this time series would indicate in-homogeneity introduced by the self-calibration or the clear sky reflection maps. The applied method is necessary in order to avoid misleading trends in the monthly differences introduced by the quite different start and end points of the BSRN station time series and the 
corresponding mismatch in the weighting of regional bias values. As can be seen in Figure 4 there is no detectable trend in the temporal evolution of the normalised bias (monthly differences), which proves evidence of the homogeneity of the SSI and CAL data for the analysed time period. In contrast, the satellite raw counts show a significant and large trend, a decrease of the sensibility due to ageing, see Figure 5. The figure shows the temporal evolution of $\rho_{\max }$ for the MVIRI period from 1993 to 2005 covering Meteosat First Generation satellites 5 to 7 . It can be seen that $\rho_{\max }$ decreases substantially with the operation time of a satellite (e.g., Meteosat 7) which reflects the degradation of the instrument with time. This degradation is corrected by the self-calibration as demonstrated by the normalised bias shown in Figure 4. The absence of a trend in the normalised bias of the SSI data demonstrates the ability of the self-calibration method to correct for ageing and satellite switches.

Figure 4. Temporal evolution of the normalised bias between the CM SAF data set and the BSRN data including the trend colored in red. The green line is the neutral axis. The blue lines at the bottom of the plot indicate the change of satellites (Meteosat-5, Meteosat-6, Meteosat-7). The slight trend is statistically not significant, hence no significant trend is apparent. The absence of a significant trend in relation to the large trends in the digital satellite counts (see Figure 5) demonstrates that the self-calibration works well.

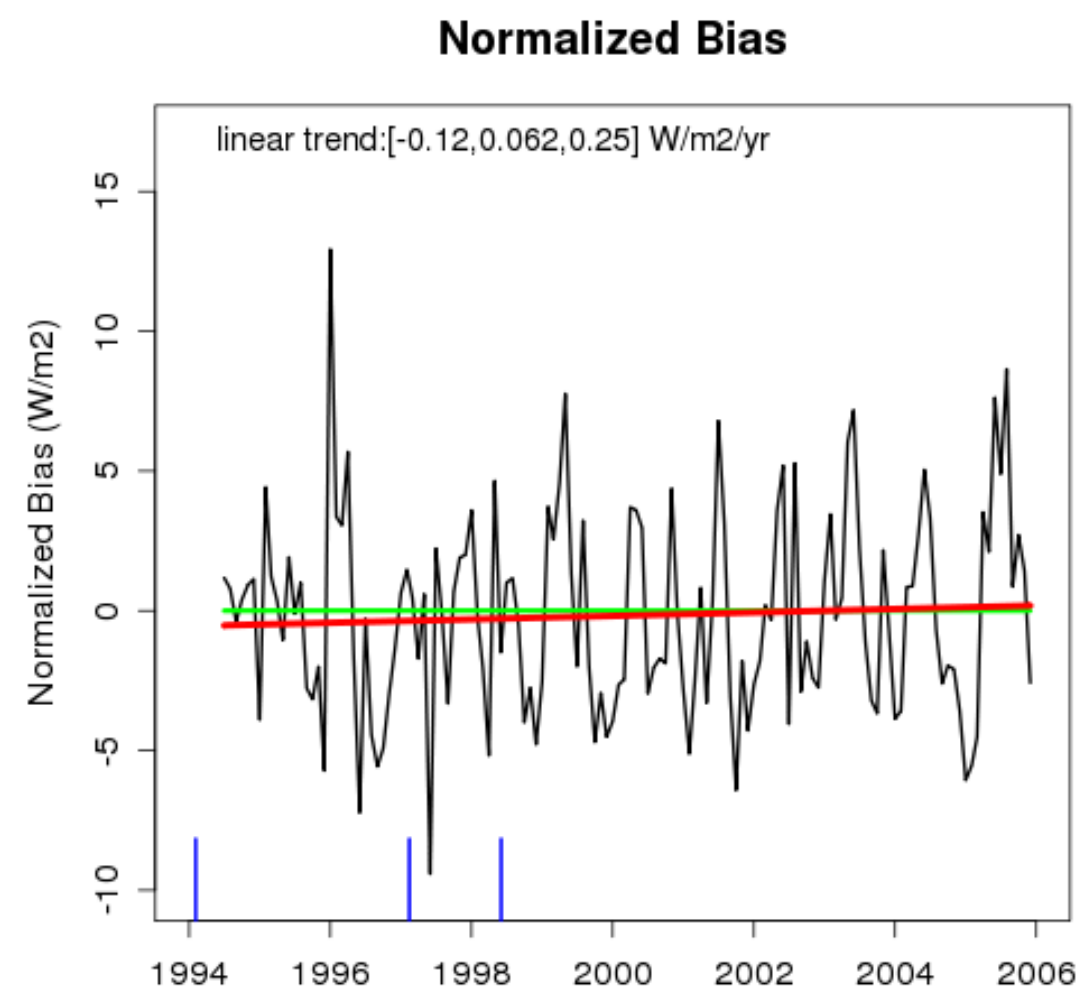

Also the Hovmöller diagrams covering the whole period (not shown here) do not show any hints for artificial trends or jumps induced by the self-calibrations method. It is worth noting that a statistically significant negative trend in the normalised differences is present in the GEWEX and ISCCP SSI data sets as illustrated in Figure 6. Concerning ISCCP our finding are in line with the results discussed by Evan et al. [21]. 
Figure 5. Temporal evolution of $\rho_{\max }$ for Meteosat-5 to Meteosat-7. A bezier fit has been applied to the $\rho_{\max }$ values.

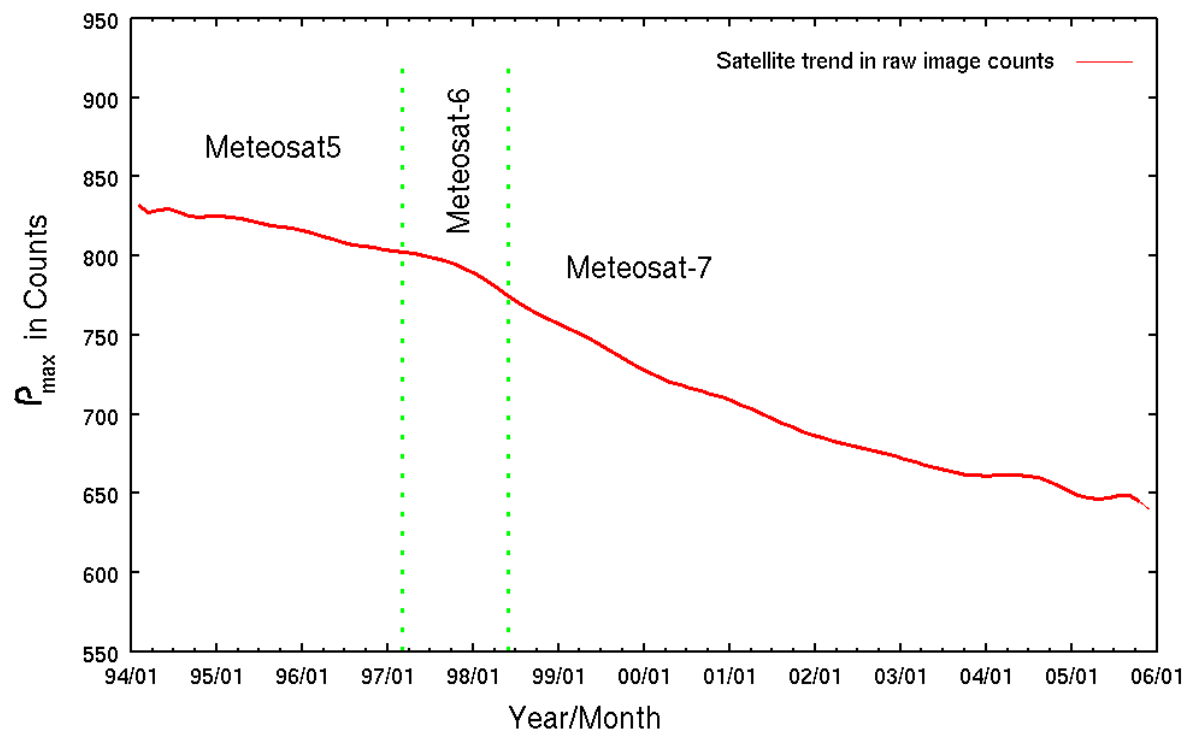

Figure 6. Temporal evolution of the normalised differences between the GEWEX and ISCCP data set relative to the BSRN data including the trend colored in red. Significant trend are apparent for both data sets indicating serious problems in the homogeneity of the data sets.

Normalized Bias (GEWEX - BSRN)

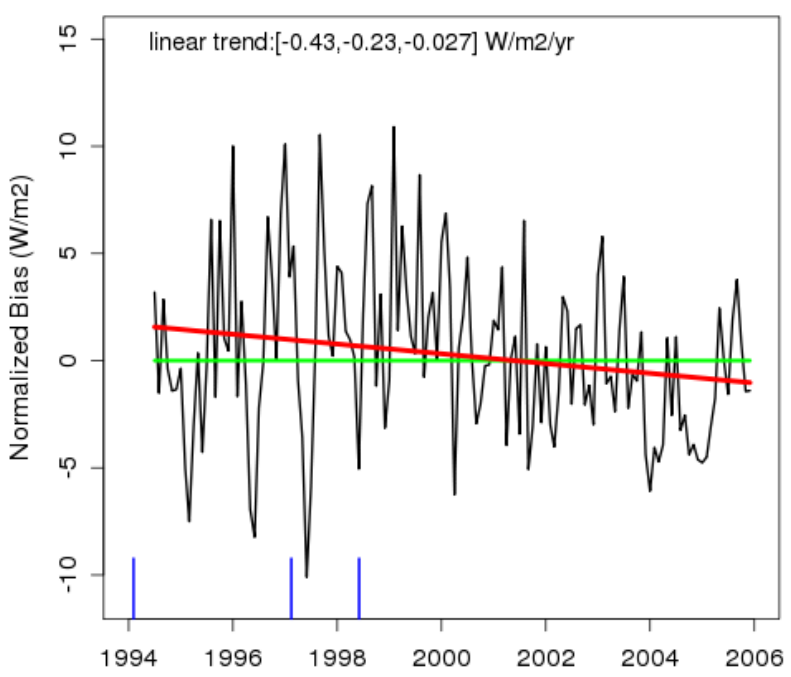

Normalized Bias (ISCCP - BSRN)

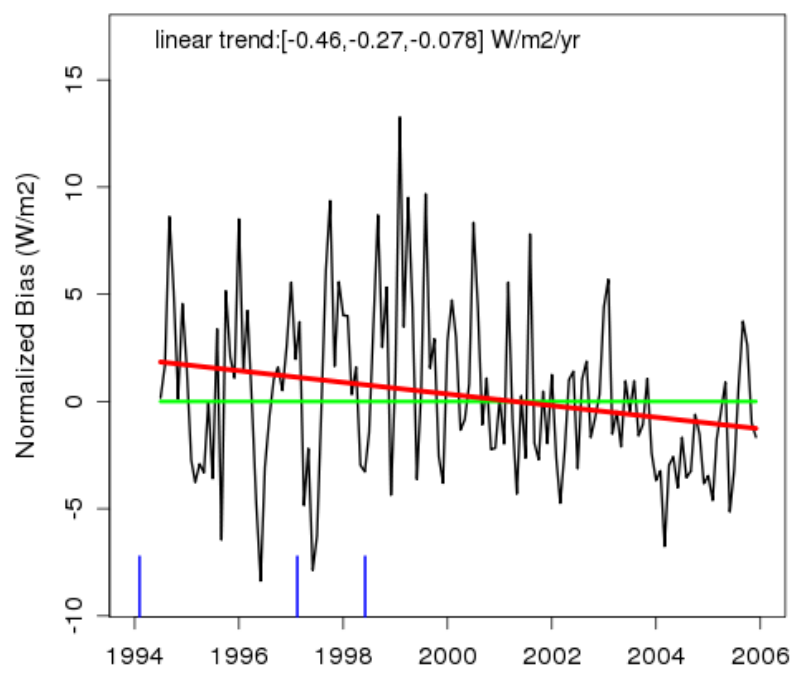

By definition the Heliosat method retrieves the effective cloud albedo. This circumvents the requirement to use land surface targets to calibrate the satellite radiances. In fact, by using a land surface target in the self-calibration method errors could be introduced as the ageing detected by observation of clear sky radiances is different than that detected with an appropriate cloud target. This effect is due to spectral dependency of the ageing of optical devices ("UV is more aggressive"), which leads to different rates of ageing for different land surface types, due to different spectral response of the targets. In our 
self-calibration method the ageing effects of the "clear sky" observations for different surface types is automatically considered by the retrieval of the clear sky reflection $\rho_{c s}$.

\section{Results and Discussion}

In order to discuss and analyse the role of the effective cloud albedo within the scope of climate monitoring and climate analysis two regions have been extracted from the CM-SAF climate data record for example trend analysis. The long term mean of the effective cloud albedo of Central Europe is shown in Figure 7. Trend analysis of the cloud albedo and the derived SIS has been performed based on R [22] using a linear model to estimate the trends illustrated in Figures 8 and 9.

Figure 7. The 23-year mean of the effective cloud albedo for central Europe.

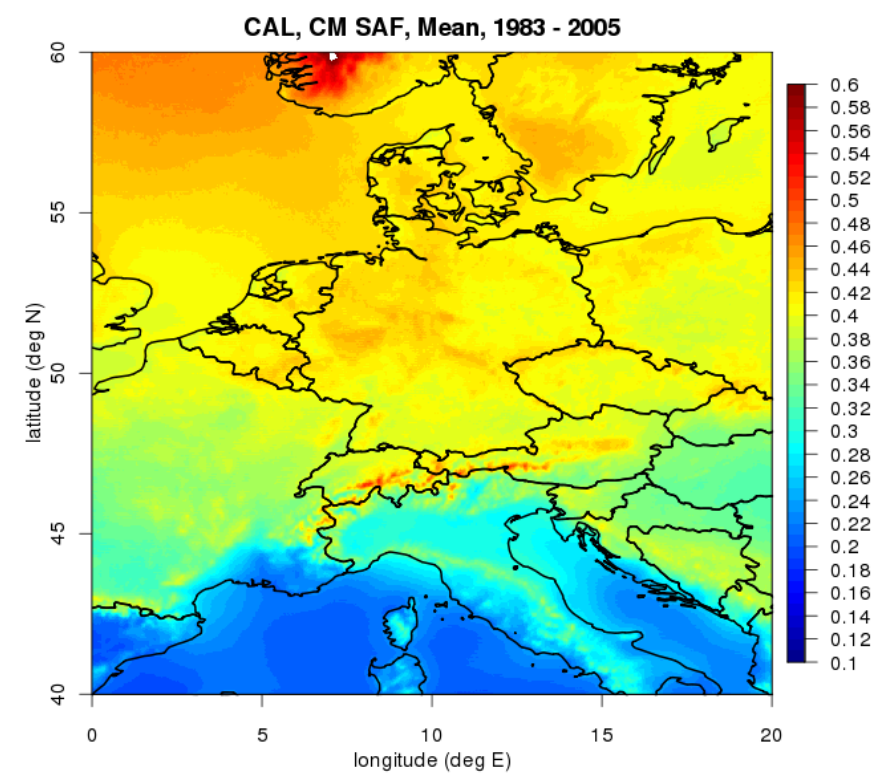

Here, it is remarkable that the trend in CAL and SIS are quite different over ocean and land. Over ocean large negative trends in CAL are apparent while over land the trends are pre-dominantly positive. This leads to positive trends over ocean and negative trends over land for the solar surface irradiance. This is also visible in other regions of the world, e.g., as illustrated in Figure 9.

Again the findings over ocean and land concerning the trend in CAL and SSI are different. CAL shows pre-dominantly a positive trend over land and an negative trend over ocean. Trends in this region are also important for the monitoring of affects induced by changes in the Hadley circulation, the driver of the Intertropical Convergence Zone (ITCZ), characterised by high cloudiness.

It has to be considered that the majority of the incoming solar irradiance is absorbed by the ocean, because of the large spatial coverage and the low surface albedo. The ocean is therefore the pre-dominant solar energy absorber and energy reservoir of the Earth. Global and regional trends in the effective cloud albedo and the derived solar surface irradiance over ocean would affect the Earth's energy system significantly on different scales. Further on, the large regional differences in the trends demonstrate the risk of using ground stations with specific spatial distribution for the monitoring and analysis of climate change. Especially, as long term records of well maintained ground based measurements are not only scarce over the ocean but also in many regions of the world, e.g., in large parts of Asia and Africa. Of 
course, this induces a high statistical uncertainty in trends of solar surface irradiance and its extremes derived from (grided) ground measurements. Trends in solar irradiance which are not accurately monitored due to missing ground measurements, could easily lead to misleading interpretations of temperature trends on a regional and global scale. These arguments apply also for the global dimming and brightening analysis and monitoring.

Figure 8. The result of the trend analysis for Central Europe. The trend in the effective cloud albedo (Top) and the trend in solar surface irradiance (SSI, (Bottom)). The regions with negative trend in CAL transfers to positive trend in SSI and visa versa. Also local differences are apparent. The Rhine valley in the South-West of Germany shows a relative large positive trend in solar irradiance. This is of importance for the wine agriculture in this region.
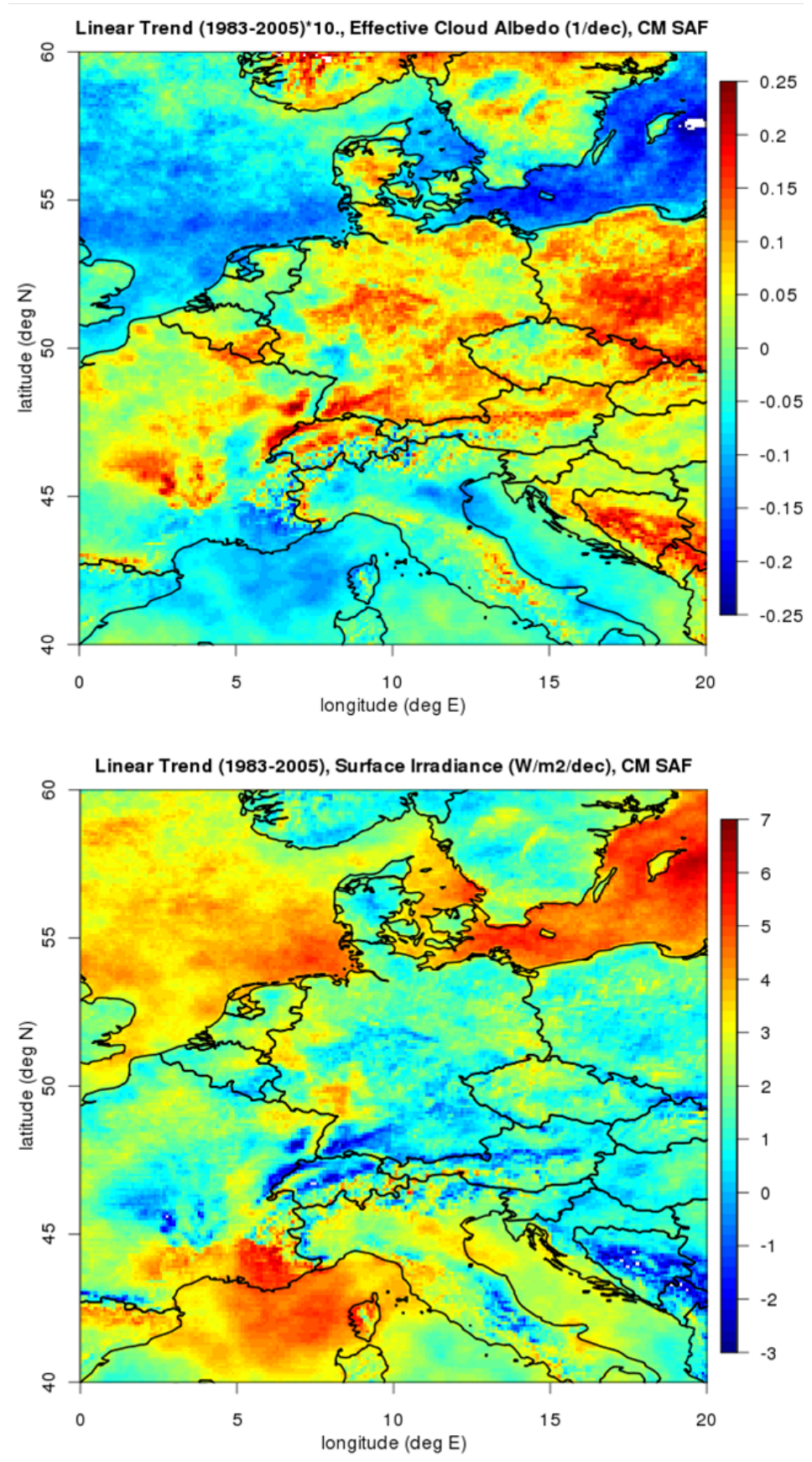
Figure 9. Trend of the effective cloud albedo and the solar surface irradiance within the ITCZ. The slightly different trends apparent in the CAL and SSI (Top, Bottom) are a result of trends in water vapour affecting SSI but not CAL.
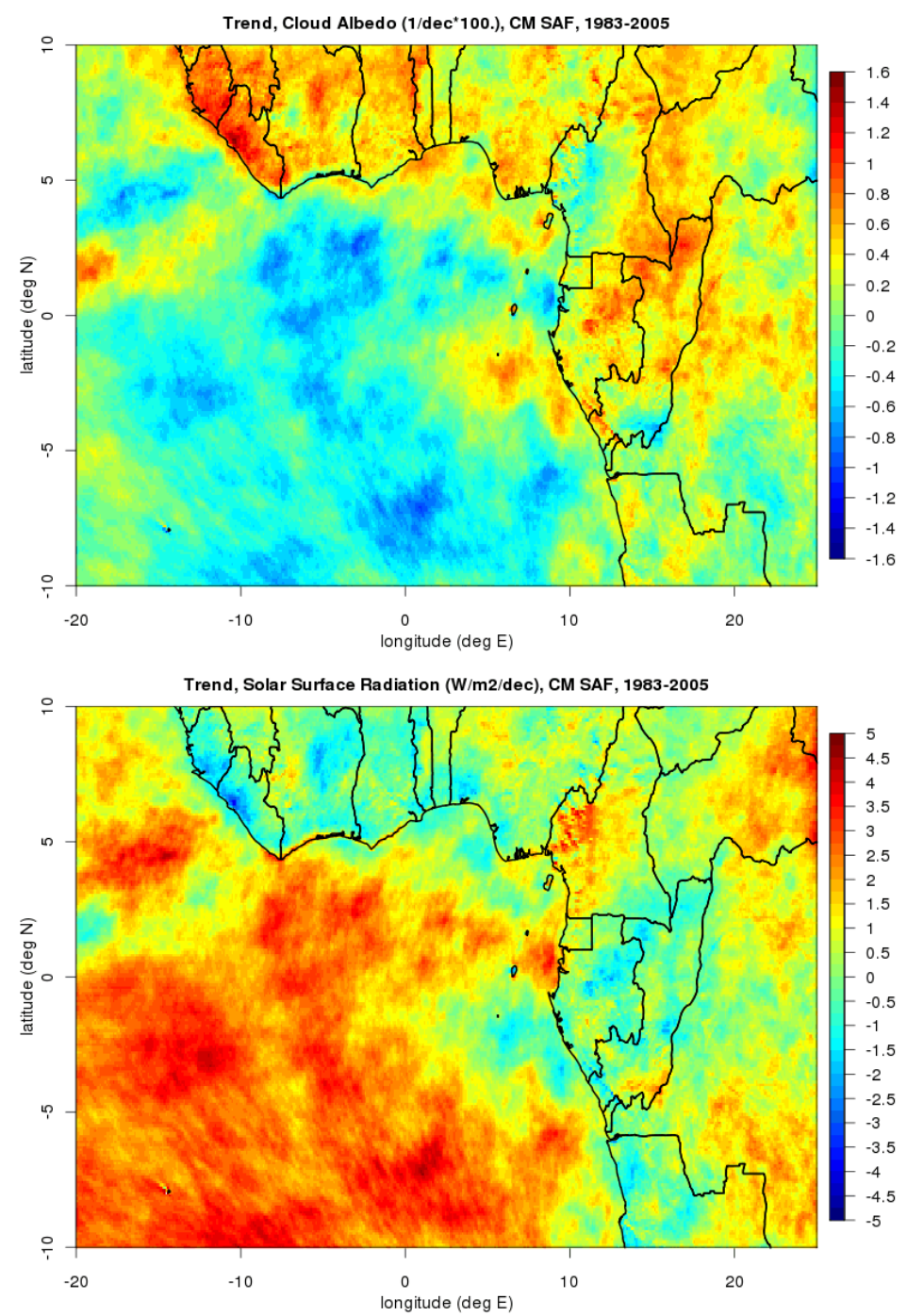

Within climate analysis global brightening and dimming has been a very important discussion, e.g., global dimming has the potential to mask the effect of global warming to some extent and brightening might lead to misleading interpretations about the magnitude of global warming induced by an increase of greenhouse gases. Wild [23] found that the various studies analyzing long-term records of solar surface irradiance suggest a widespread decrease in solar radiation between the 1950s and 1980s (global dimming), with a partial recovery after the dimming period at many locations (brightening). Changes in the aerosol load have been proposed as the main driver for the dimming and brightening. However, the majority of research directed to the analysis of dimming and brightening has been based on ground measurements [23], which are pre-dominantly located on land. The trends in the satellite-derived effective cloud albedo and solar surface irradiance given in Figures 8 and 9 show opposite trends over ocean and land, which demonstrates the need to include the ocean within dimming and brightening studies in order to avoid misleading interpretations. This can be done by the use of accurate satellite derived data sets. Recently, satellite data (partly in combination with ground based 
stations) have been used to overcome this problem induced by using pre-dominantly only ground based measurements [24,25]. Within these studies GEWEX and ISCCP satellite-based data have been used. Pinker et al. [25] found that the global-scale findings of dimming/brightening differ in sign and magnitude from previously reported ground observations. Also over the land surface, Wild [23] and Pinker et al. [25] disagree. However, as illustrated in Figure 6 artificial trends in the ISCCP and GEWEX satellite-based solar surface irradiance induces a significant uncertainty and make final conclusions difficult. The Heliosat method, in particular the self-calibration method, discussed in this manuscript has been proven to be able to provide a homogenous and stable solar surface irradiance data record derived from the effective cloud albedo. Hence, the respective climate data records are expected to be quite useful in order to get a more complete and more reliable picture about trends in solar surface irradiance and the effective cloud albedo.

Another aspect, the effective cloud albedo enables the analysis of causes of trends in the solar surface irradiance and a clear separation between direct aerosol effect and cloud effect. Within this scope the effective cloud albedo can be used to investigate the indirect aerosol effect.

\section{Summary and Conclusions}

GCOS defines cloud properties and the Earth's radiation budget as essential climate variables. The cloud albedo is a measure for the portion of radiation reflected back to space by clouds. It has been shown that this information is essential for the analysis and interpretation of the Earth's radiation budget and the solar surface irradiance. A method for the generation of the effective cloud albedo and the solar surface irradiance based on the visible channel $(0.45-1 \mu \mathrm{m})$ of the MVIRI instruments on-board the first generation of Meteosat satellites has been presented and discussed. This method includes a newly developed self-calibration approach and has been used to generate a 23 year long (1983-2005) continuous and validated climate data record of the effective cloud albedo and solar surface irradiance. The ability of the method to provide these essential climate variables in high accuracy and homogeneity has been demonstrated by comparison with BSRN measurements. We found opposite trends in the observed effective cloud albedo over land and ocean resulting in positive trends in the solar surface irradiance and partly negative trends over land. Over ocean ground measurements of solar irradiance and cloud properties are almost not available. It has been shown that satellite-derived effective cloud albedo and solar surface irradiance constitutes a powerful observational data source in order to complement ground-based measurements. Within this scope it has to be considered that the ocean is the main energy reservoir of the Earth which emphasis the role of satellite-observed effective cloud albedo an derived solar surface irradiance. Further on, the large regional differences in the trends demonstrate the risk of using ground stations with specific spatial distribution for the monitoring and analysis of climate change. Especially, as long term records of well maintained ground based measurements are not only scarce over the ocean but also in many regions of the world, e.g. in large parts of Asia and Africa. Of course, this induces a high statistical uncertainty in trends of solar surface irradiance and its extremes derived from (gridded) ground measurements. Trends in solar irradiance which are not accurately monitored due to missing ground measurements, could easily lead to misleading interpretations of temperature trends on a regional and global scale. These arguments apply also for global dimming and brightening analysis and monitoring. It has been shown that homogenous time series of satellite-derived effective cloud albedo 
and solar surface irradiance provide essential information for better analysis of global brightening and dimming, which is in turn important for the analysis and interpretation of climate change. Therefore, we see the need to include the analysis of the effective cloud albedo within brightening and dimming studies and interpretations of regional temperature trends.

There is a need for accurate solar surface irradiance for regional climate monitoring as well as for solar energy applications. We have shown that solar surface irradiance derived from satellite-observed effective cloud albedo is a reliable source characterised by high accuracy and homogeneity. The satellite-observed effective cloud albedo and the derived solar surface irradiance are essential to complete the global climate observation system. Hence, we see a urgent need to consider these quantities more explicitly within the GCOS mission and frame.

\section{Acknowledgements}

This work is partly funded by EUMETSAT within the SAF framework. We thank the European tax payers, as the funding is originally given by them. Thanks to BSRN for the ground based-data.

\section{References}

1. Harrison, E.F.; Barkstrom, B.R.; Ramanathan, V.; Cess, R.D.; Gibson, G.G. Seasonal variation of cloud radiative forcing derived from the Earth Radiation Budget Experiment. J. Geophys. Res. 1990, 95, 18.687-18.703.

2. Trenberth, K.; Fasulo, J.; Kiehl, J. Earth's global energy budget. Bull. Amer. Meteor. Soc. 2009, 90, 311-323.

3. Liu, Y.; Wu, W.; Jensen, M.; Toto, T. Relationship between cloud radiative forcing, cloud fration and cloud albedo, and new surface-based approach for determining cloud albedo. Atmos. Chem. Phys. Discuss. 2011, 11, 5681-5698.

4. Ramanthan, R.; Cess, R.A. Cloud radiative forcing and climate: Results from the Earth Radiation Budget Experiment. Science 1989, 243, 57-63.

5. Zhang, Y.C.; Rossow, W.B.; Lacis, A.A. Calculation of surface and top of atmosphere radiative fluxes from physical quantities based on ISCCP data sets: 1. Method and sensitivity to input data uncertainties. J. Geophys. Res. 1995, 100, 1149-1165.

6. Cox, S.; Gupta, S.; Mikovitz, J.; Chiacchio, M.; Zhang, T.; Stackhouse, P. The NASA/GEWEX Surface Radiation Budget Dataset: Results and Analysis. In Proceedings of the International Radiation Symposium, Busan, Korea, 23-28 August 2004; In IRS 2004: Current Problems in Atmospheric Radiation; Fischer, H., Sohn, B.J., Eds.; A. Deepak Publishing: Hampton, VA, USA; 2004; pp. 419-422.

7. Rossow, W.B.; Zhang, Y.Z. Calculation of surface and top of atmosphere radiative fluxes from physical quantities based on ISCCP data sets: 2. Validation and first results. J. Geophys. Res. 1995, 100, 1167-1197.

8. Rossow, W.B. Measuring cloud properties from space: A review. J. Climate 1989, 2, 201-213.

9. Stubenrauch, G.; Kinne, S.; GEWEX-team. Assessment of global cloud climatologies. GEWEX News February 2009; Volume 19, pp. 6-7. 
10. Wielicki, B.A.; Wong, T.; Loeb, N.; Minnis, P.; Priestley, K.; Kandel, R. Changes in Earth's albedo measured by satellite. Science 2005, doi:10.1126/science.1106484.

11. Posselt, R.; Mueller, R.; Stöckli, R.; Trentmann, J. Spatial and temporal homogeneity of solar surface irradiance across satellite generations. Remote Sens. 2011, 3, 1029-1046.

12. Cano, D.; Monget, J.M.; Albuisson, M.; Guillard, H.; Regas, N.; Wald, L. A method for the determination of the global solar-radiation from meteorological satellite data. Solar Energy 1986, $37,31-39$.

13. Beyer, H.G.; Costanzo, C.; Heinemann, D. Modifications of the Heliosat procedure for irradiance estimates from satellite images. Solar Energy 1996, 56, 207-212.

14. Hammer, A.; Heinemann, D.; Hoyer, C.; Kuhlemann, R.; Lorenz, E.; Müller, R.; Beyer, H. Solar energy assessment using remote sensing technologies. Remote Sens. Environ. 2003, 86, 423-432.

15. Eumetsat. The Meteosat Archive User Handbook; Technical Report; Eumetsat: Darmstadt, Germany, 2001.

16. Eumetsat. Meteosat First Generation. Available online: http://www.eumetsat.int/Home/Main/ Satellites/MeteosatFirstGeneration (accessed on 20 October 2011).

17. Mueller, R.; Matsoukas, C.; Gratzki, A.; Behr, H.; Hollmann, R. The CM-SAF operational scheme for the satellite based retrieval of solar surface irradiance-A LUT based eigenvector hybrid approach. Remote Sens. Environ. 2009, 113, 1012-1024.

18. Ohmura, A.; Dutton, E.G.; Forgan, B.; Fröhlich, C.; Gilgen, H.; Hegner, H.; Heimo, A.; Konig-Langlo, G.; McArthur, B.; Müller, G.; Philipona, R.; Pinker, R.; Whitlock, C.H.; Dehne, K.; Wild, M. Baseline Surface Radiation Network (BSRN/WCRP): New precision radiometry for climate research. Bull. Amer. Meteor. Soc. 1998, 79, 2115-2136.

19. Raschke, E.; Bakan, S.; Kinne, S. An assessment of radiation budget data provided by ISCCP and GEWEX-SRB. Geophys. Res. Lett. 2006,, doi:10.1029/2005GL025503.

20. Rigollier, C.; Lefevre, M.; Blanc, P.; Wald, L. The operational calibration of images taken in the visible channel of the meteosat series of satellites. J. Atmos. Ocean. Technol. 2002, 19, 1285-1293.

21. Evan, A.T.; Heidinger, A.K.; Vimont, D.J. Arguments against a physical long term trend in ISCCP cloud amounts. Geophys. Res. Lett. 2007, doi:10.1029/2006GL028083.

22. Adler, J. R In a Nutshell; O’Reilly Media: Sebastopol, CA, USA, 2010.

23. Wild, M. Global dimming and brightening: A review. J. Geophys. Res. 2009, doi:10.1029/2008JD011470.

24. Hinkelmann, L.M.; Stackhouse, P.W.; Wielicki, B.A.; Zhang, T.; Wilson, S.R. Surface insolation trends from satellite and ground measurements: Comparison and challenges. J. Geophys. Res. 2009, doi:10.1029/2008JD011004..

25. Pinker, R.T.; Zhang, B.; Dutton, E.G. Do satellites detect trends in surface solar radiation? Science 2005, 308, 850-854.

(C) 2011 by the authors; licensee MDPI, Basel, Switzerland. This article is an open access article distributed under the terms and conditions of the Creative Commons Attribution License (http://creativecommons.org/licenses/by/3.0/.) 